Article

\title{
Admissible Perturbation of Demicontractive Operators within Ant Algorithms for Medical Images Edge Detection
}

\author{
Cristina Ticala * and Ioana Zelina and Camelia-M. Pintea \\ Technical University of Cluj-Napoca, North University Center of Baia Mare, 400114 Cluj-Napoca, Romania; \\ ioanazelina@yahoo.com (I.Z.); dr.camelia.pintea@ieee.org (C.-M.P.) \\ * Correspondence: cristina.ticala@cunbm.utcluj.ro
}

Received: 24 May 2020; Accepted: 23 June 2020; Published: 26 June 2020

check for updates

\begin{abstract}
Nowadays, demicontractive operators in terms of admissible perturbation are used to solve difficult tasks. The current research uses several demicontractive operators in order to enhance the quality of the edge detection results when using ant-based algorithms. Two new operators are introduced, $\chi$-operator and $\mathrm{KH}$-operator, the latter one is a Krasnoselskij admissible perturbation of a demicontractive operator. In order to test the efficiency of the new operators, a comparison is made with a trigonometric operator. Ant Colony Optimization (ACO) is the solver chosen for the images edge detection problem. Demicontractive operators in terms of admissible perturbation are used during the construction phase of the matrix of ants artificial pheromone, namely the edge information of an image. The conclusions of statistical analysis on the results shows a positive influence of proposed operators for image edge detection of medical images.
\end{abstract}

Keywords: fixed point; Krasnoselskij iteration; admissible perturbation; Ant Colony Optimization

\section{Introduction}

Admissible perturbation theory is a direction of research which unifies the main aspects of iterative approximating. The theory of admissible perturbations of an operator introduced by Rus in [1] opens a new direction of research and unifies major aspects of the iterative approximation of fixed point for single valued self operators. The admissible perturbation of a nonlinear operator was studied in detail in [2] and extended for the generalized pseudocontractive operators by Berinde, Khan and Fukhar-ud-din [3].

Demicontractive operators are still a very recent object of study [4-6]. In [6] the authors solve split common fixed point problems for demicontractive mappings in Hilbert spaces as an extension of the Halpern type algorithm from [7] to a viscosity iteration. In [8] are shown the approximated solutions for variational inequalities in terms of admissible perturbations. Furthermore, in [9] a weak convergence theorem in Hilbert spaces using admissible perturbations is introduced. A standard approach for admissible perturbations of demicontractive operators is provided in [10].

Difficult problems needs highly-efficient algorithms to obtain feasible results. Nowadays, Ant Colony Optimization (ACO) [11,12] is a successfully probabilistic technique for solving complex problems as routing, assignment, scheduling problems [13-16] and some generalized versions as [17-19]. In 2019 Chowdhury did a case study with drones for wildlife surveillance [20].

In order to solve the image edge detection, a problem of identifying discontinuities in brightness and therefore enhancing edges of objects in an image, different ant algorithms were involved during last years. From the most recent ones, Shi et al. [21] with Canny Edge and ACO, make use of a fuzzy triangle membership function in the neighborhood of the gray value, and Mahalingam and Subramoniam [22] used ACO for an optimized object detection and tracking. 
In [23] ACO is implemented by the memristor crossbar array and uses parallel computing; threshold generates edges as mean of the final conductance matrix; the results are comparatively better than neural networks models and while using Sobel operator. A recent survey on nature-inspired medical image in biomedical data [24] includes the behavior of swarm intelligence. In order to combine capabilities of several independent exact or meta-heuristics, an integrative cooperative search framework was proposed in [25].

As a part of swarm intelligence heuristics categories, ant colonies algorithms are inspired by real ants' behavior in finding shortest paths while using as information the trail of a chemical substance, deposited by ants, called pheromone. Ants pheromone sensitivity was proposed in [26,27]; in order to process medical images, Pintea and Ticala proposed the first theoretical ACO approach involving pheromone sensitivity in [28].

A heuristic value making use of two admissible perturbation operators was applied in [29] to a demicontractive mapping in order to solve the image edge detection problem with ACO. The current work makes a step forward and uses admissible perturbations of demicontractive mappings as test functions. The agents have different roles in the image edge detection: some are explorers, and others exploiters; in time they change their roles based on the environment dynamics.

The paper is further structured as follows. Methodologies including a preview of the theory of admissible perturbations and the Ant Colony Optimization are described in Section 2. The case study of the ACO algorithm for edge detection in medical images, with all processes involved from initialization to the decision follows in Section 3. Further, Section 4 includes: experimental results and comparison of ant-based algorithms and operators involved, the representation of results medical images, statistical analysis and final discussions. The paper concludes in a positive manner, as the proposals of the current work obtain promising results for the tested set of the medical images.

\section{Methodology}

\subsection{Theory of Admissible Perturbations}

Rus proposed in [1] the theory of admissible perturbations of an operator and succeeded to unify iterative approximation of fixed point for single valued self operators. The following are the main related definitions and specific examples.

Definition 1. The operator $T: C \rightarrow C$ is demicontractive if there exists a constant $k<1$ such that, for each fixed point $p$ of $T$ and each $x \in C$, the following inequality is true [30].

$$
\|T x-p\|^{2} \leq\|x-p\|^{2}+k\|x-T x\|^{2} .
$$

The constant $k$ from Equation (1) is called the contraction coefficient.

Example 1. An example of demicontractive mapping is $T: \mathbb{R} \rightarrow \mathbb{R}$, defined by Equation (2) as in [31].

$$
T x=\left\{\begin{array}{l}
\frac{2}{3} x \sin \frac{1}{x}, \text { if } x \neq 0 \\
0, \text { if } x=0
\end{array}\right.
$$

Definition 2. Let $X$ be a nonempty set. A mapping $G: X \times X \rightarrow X$ is called admissible if it satisfies the following two conditions, (A1) with Equation (3) and (A2) with Equation (4) as in [1].

$$
\begin{aligned}
& (A 1): G(x, x)=x, \text { for all } x \in X \\
& (A 2): G(x, y)=x, \text { implies } y=x .
\end{aligned}
$$


Definition 3. Let $f: X \rightarrow X$ be an operator. Then the admissible mapping $f_{G}: X \rightarrow X$ defined by Equation (5) is called the admissible perturbation of $f$.

$$
f_{G}(x):=G(x, f(x)),
$$

Definition 4. Let $f: X \rightarrow X$ be a nonlinear operator and $G: X \times X \rightarrow X$ be an admissible mapping. The iterative algorithm $\left\{x_{n}\right\}_{n \in \mathbb{N}}$ given by Equation (6), as in [1], is called the Krasnoselskij algorithm corresponding to $G$, or the GK-algorithm.

$$
\left\{\begin{array}{l}
x_{0} \in X, \\
x_{n+1}=G\left(x_{n}, f\left(x_{n}\right)\right), n \geq 0
\end{array}\right.
$$

Example 2. Let $(V,+, \mathbb{R})$ be a real vector space, $X \subset V$ a convex subset, $\lambda \in(0,1)$ and $G: X \times X \rightarrow X$ defined by Equation (7), then $f_{G}$ is an admissible perturbation of $f$, further called the Krasnoselskij perturbation of $f$.

$$
G(x, y):=(1-\lambda) x+\lambda y, x, y \in X
$$

Example 3. Let $(V,+, \mathbb{R})$ be a real vector space, $X \subset V$ a convex subset, $\chi: X \times X \rightarrow(0,1)$ a mapping and $G: X \times X \rightarrow X$ defined by Equation (8), then $f_{G}$ is an admissible perturbation of $f$.

$$
G(x, y):=(1-\chi(x, y)) x+\chi(x, y) y
$$

We recall that an admissible perturbation is an operator with the properties (A1) Equation (3) and (A2) Equation (4). Admissible perturbations are thoroughly studied in respect to different types of operators.

\subsection{Ant Colony Optimization for Edge Detection Using Proposed Operators}

From meta-heuristics we choose Ant Colony Optimization (ACO) which keeps high standards for solving complex problems including images edge detection. ACO builds optimal solution of the target problem using artificial ants while searching the best path in the search space. The ants deposits pheromones during this process. The "memory" of ants is the artificial pheromone trail, which is updated during the search process.

The quality of a partial solution is given by the amount of pheromone modified along the way. The global solution is found after all ants, based on the previous pheromone information, are guided to more promising regions in the search space. For the $K$ ants engaged in the search, in a space $\mathcal{X}$, with $M_{1} \times M_{2}$ nodes, $\mathrm{ACO}$ is summarized as follows:

- $\quad$ Initiate all $K$ ants, the pheromone matrix, $\tau^{(0)}$.

- For every solution construction step index $n=1: N$

- $\quad$ For every ant $k=1: K$ successively move the $k$-th ant for $L$ movement steps based on the transition probability matrix, Equation (9).

- $\quad$ Update the pheromone matrix $\tau^{(n)}$, Equation (10).

- Make the solution decision based on the final pheromone matrix $\tau^{(N)}$.

There are two fundamental problems which ACO has to solve, and will be further detailed.

- $\quad$ At first, ACO will build the transition probability matrix. $p^{(n)}$.

- Secondly, ACO will update the pheromone matrix $\tau^{(n)}$.

At the $n$-th construction step, $k$-th ant moves from node $I$ to $J$ (Equation (9)), where $\tau_{I J}$ is the pheromone value of $\operatorname{arc}(I, J)$. 
The heuristic value of $(I, J)$ with the same value for all the $N$ construction steps is $\eta_{I J}$; the weighting factors for pheromone and heuristic are $\alpha, \beta$, and the neighborhood of node $I$ is $\Omega_{I}$.

$$
p_{I J}^{n}=\frac{\left(\tau_{I J}^{(n-1)}\right)^{\alpha}\left(\eta_{I J}\right)^{\beta}}{\sum_{J \in \Omega_{I}}\left(\tau_{I J}^{(n-1)}\right)^{\alpha}\left(\eta_{I J}\right)^{\beta^{\prime}}} \quad \text { if } J \in \Omega_{I},
$$

The information in the pheromone matrix is updated twice during ACO. First, a local update is performed after each ant moves within each construction step, the second, global update is made after the move of all $K$ ants within each construction step.

Local update. The local update of the pheromone matrix is performed after the move of the $k$-th ant in the $n$-th construction step as in Equation (10).

$$
\tau_{I J}^{(n)}= \begin{cases}\tau_{I J}^{n-1} \cdot(1-\rho)+\rho \cdot \Delta_{I J}, & \text { if }(I, J) \text { is on } \\ \tau_{I J}^{(n-1)}, & \text { the best tour } \\ \text { otherwise. }\end{cases}
$$

In Equation (10) $\rho$ is the pheromone evaporation rate, $\Delta_{I J}$ is the quantity of pheromone laid on edge $(I, J)$. The best tour is considered the best tour found in a current step of an iteration.

Global Update. The exploration is enhanced to further obtain better global and best solution. The global update uses Equation (11). The pheromone decay coefficient is denoted by $\psi$.

$$
\tau^{(n)}=(1-\psi) \cdot \tau^{(n-1)}+\psi \cdot \tau^{(0)},
$$

\section{Case Study: ACO Algorithm for Edge Detection in Medical Images}

The proposed edge detection algorithm uses artificial ants which move in a bi-dimensional image in order to build the pheromone matrix, each element of the matrix represents the edge information for every pixel in the image. The new ant technique starts with the initialization process, runs for $N$ construction steps when it creates and updates pheromone matrix and at last, performs the decision process to determine the edge.

- Initialization process. In the initialization process, all the $K$ ants are placed randomly on the image. Each pixel of the image is viewed as a node. Each value of the initial pheromone matrix $\tau^{(0)}$ is set to a constant $\tau^{\text {init }}$. A constant value $L$ used to define the number of movement steps in the construction process is defined.

- Construction process. At the $n$-th construction step, one ant is randomly chosen from all $K$ ants, this ant will be consecutively moving for $L$ movement steps. The ant will move from node $i$ to $j$ based on the transition probability, Equation (9).

There are two main aspects in the construction process.

1. First, it is the issue of establishing the heuristic $\eta_{i j}$ from Equation (9).

2. The second issue is to establish the domain in which one ant found in node $(l, m)$ can make moves, i.e., $\Omega_{l, m}$ from Equation (9).

The connectivity domain is based on the matrix notation of neighbors nodes: the 8-connectivity domain, the eight closest neighbors of $I_{i j}$ gray square from Figure 1. We propose to compute the heuristic value based on the local statistic of the pixel $(i, j)$, Equation (12) where $Z=\sum_{i=1}^{M_{1}} \sum_{j=1}^{M_{2}} V_{c}\left(I_{i, j}\right)$, is a normalization factor, $I_{i, j}$ is the intensity value of the pixel at the position $(i, j)$ from the image, the function $V_{c}\left(I_{i, j}\right)$ is a function which processes $c I_{i, j}$ called "clique", defined in [32], as in Figure 1.

$$
\eta_{i, j}=\frac{1}{Z} \cdot V_{c}\left(I_{i, j}\right)
$$




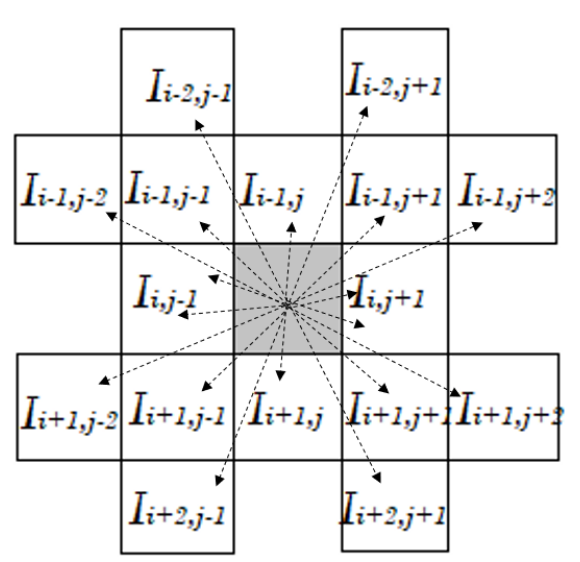

Figure 1. Local configuration at $I_{i, j}$ pixel, $c I_{i, j}$, for computing the variation value $V_{\mathcal{c}}\left(I_{i, j}\right)$ defined by Equation (13). The pixel $I_{i, j}$ is the gray square.

The value of $V_{c}\left(I_{i, j}\right)$ depends on the variation of image's intensity values on $c I_{i, j}$. More specifically, the value of $V_{c}\left(I_{i, j}\right)$ at the pixel $I_{i, j}$ is considered in Equation (13).

$$
\begin{aligned}
V_{c}\left(I_{i, j}\right)= & f\left(\left|I_{i-2, j-1}-I_{i+2, j+1}\right|+\left|I_{i-2, j+1}-I_{i+2, j-1}\right|\right. \\
& +\left|I_{i-1, j-2}-I_{i+1, j+2}\right|+\left|I_{i-1, j-1}-I_{i+1, j+1}\right| \\
& +\left|I_{i-1, j}-I_{i+1, j}\right|+\left|I_{i-1, j+1}-I_{i+1, j-1}\right| \\
& \left.+\left|I_{i-1, j+2}-I_{i+1, j-2}\right|+\left|I_{i, j-1}-I_{i, j+1}\right|\right) .
\end{aligned}
$$

As prerequisites for operators, $\chi: \mathbb{R} \times \mathbb{R} \longrightarrow[0,1)$, is defined as in Equation (14) and $y: \mathbb{R} \rightarrow \mathbb{R}$ as in Equation (15).

$$
\begin{aligned}
& \chi(x, y)=\frac{x^{2} \cdot y^{2}}{\left(1+x^{2}\right) \cdot\left(1+y^{2}\right)} . \\
& y(x)=\left\{\begin{array}{l}
\frac{2}{3} x \sin \frac{1}{x}, \text { if } x \neq 0 \\
0, \text { if } x=0
\end{array}\right.
\end{aligned}
$$

The considered operators for $f(\cdot)$ from Equation (13) are illustrated in Figure 2 and further detailed.

$$
\begin{gathered}
f(x)= \begin{cases}\sin \left(\frac{\pi x}{2 \lambda}\right), & 0 \leq x \leq \lambda \\
0, & \text { otherwise. }\end{cases} \\
f(x)= \begin{cases}(1-\lambda) \cdot x+\lambda \cdot \frac{2}{3} x \sin \frac{1}{x} & \text { if } x \neq 0 \\
0, & \text { if } x=0 .\end{cases} \\
f(x)= \begin{cases}(1-\chi(x, y(x))) \cdot x+\chi(x, y(x)) \cdot y(x) & \text { if } x \neq 0 \\
0 & \text { if } x=0 .\end{cases}
\end{gathered}
$$

The parameter $\lambda$ from Equation (16) adjusts the shape of the operators. This operator is the same test function used in [32]. The research on admissible perturbations of demicontractive operators, by using Krasnoselskij perturbation on the demicontractive mapping Equation (2), lead us to define the $\mathrm{KH}$-operator, Equation (17). The proposed $\mathrm{KH}$-operator can be used in the same way as the other operators when constructing heuristic value during ACO. 

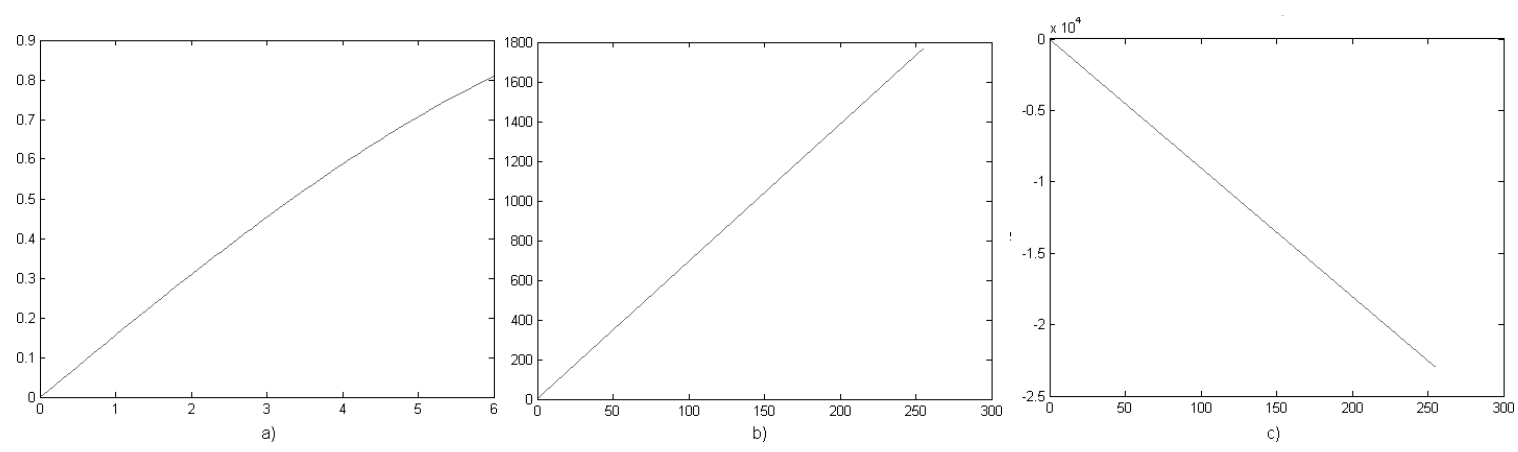

Figure 2. Operators' representations: (a) the function defined in Equation (16), $\operatorname{Sin}$, (b) the function defined in Equation (17), KH-operator, and (c) the function defined in Equation (18), Chi, $\chi$-operator.

Another proposal included is the admissible perturbation operator, the Chi, $\chi$-operator based on [1] and described by Equation (7). The Chi, $\chi$-operator from Equation (18) is an admissible perturbation operator obtained by applying the function $\chi$ defined by Equation (14), to the demicontractive mapping, Equation (2).

- Update process. The algorithm uses two update operations for the pheromone matrix.

- First update is made as in Equation (10) after each ant moves within each construction step. Each element of the matrix is updated.

- The second update is made after the movement of all ants within each construction step, Equation (11).

\section{- Decision process}

At this point of the algorithm, a binary decision is made for each pixel in order to establish if it is edge or not, by applying a threshold $T$ on the final pheromone matrix $\tau^{(N)}$. The threshold is computed as stated in [33].

\section{Results and Discussion}

The section includes description of the experiments in Section 4.1, representation of the results in Section 4.2, statistical analysis Section 4.3 and related discussions about ant-based algorithm.

\subsection{Experimental Results}

Experimental work for is further described in detail.

A. Preliminary settings for Ant Colony Optimization:

- Data-set. The experimental data set includes the medical images for the experiments as in Figure 3. The current study makes use of four medical images, as shows Figure 3. The medical image Brain CT with $128 \times 128$ resolution could be provided for free by request from authors for scientific reasons; Hand X-ray from [34] with original $225 \times 225$ resolution was reduced to $128 \times 128$ resolution to make a valid comparison with the other images, as Head CT from [35] has originally also $128 \times 128$ resolution; these two medical-images are available online for free.

- Software used. The ACO-based edge detection approach was implemented using MatLab and run on a computer with an AMD Rysen 5 2500U, 2GHz processor.

- Parameter settings for Ant Colony Optimization.

The majority of parameters values used are based on [32]. Figure 4 illustrates the way we tested the parameters: $\beta \in\{0.1,0.6,1.2\}$ and $\rho \in\{0.1,0.6,1.0\}$ for $\operatorname{Sin}, K H$ and Chi operators 
on all data-set of medical images: Brain CT, Hand X-ray and Head CT. As a result we used the best parameters found during tests.

\section{- Parameters considered for Ant Colony Optimization}

- the number of ants is based on the dimension of the image $K=\lfloor\sqrt{M 1 \times M 2}\rfloor$ : where $\lfloor$ and $\rfloor$ are the left and right rounded values to the nearest integers less than or equal to $x$; for the particular case of the $128 \times 128$ image resolution, 128 is the number of ants.

- each ant makes 300 movements in each of the $L=100$ steps; therefore in the particular case of 128 ants, 38,400 movements are made during each of the $L$ steps.

- the connectivity neighborhood $\Sigma=8$ is based on the ant's movement range in Equation (9);

- the value of each component of the pheromone matrix, $\tau_{\text {init }}=0.0001$;

- weighting factors of the pheromone information, $\alpha=1$ and heuristic information, $\beta=0.1$, in Equation (9);

- the evaporation rate, $\rho=0.1$, Equation (10);

- the pheromone decay coefficient, $\psi=0.001$, Equation (11);

- the adjusting factor of the functions, $\lambda=10$, as in Equations (16)-(18);

- $\quad$ tolerance $\varepsilon=0.1$ used in the decision process of the proposed method.

- termination criteria is given by reaching the maximal number of steps $L$.

B. Numerical results and running time:

- ACO Numerical Results. Table 1 shows that more pixels are correctly identified on the edge of the image, more precise is the edge detection of an medical image. The results are decimal scaled, and are standard values of correctly identified edges with values in the unit interval for an accurate visibility.

- ACO Running Time. Based on the computing characteristics, the average running time was 4500 seconds for each medical image. Furthermore, the running time increases as Denoise Convolutional Neural Network (DnCNN) to enhance each image as follows.

C. Software:

- We make use of the Tian et al. [32] software from the 2008 CEC conference, an image edge detection using Ant Colony Optimization MatLab software [36]. The existing software was at first modified for the use of the operators from [32] and described in Equation (16) (Sin-operator) and the proposed operators given in Equation (17) (KH-operator) and Equation (18) (Chi-operator).

- Denoise Convolutional Neural Network (DnCNN) a pretrained network [37] is used to improve the quality of the resulting medical image. Image Processing Toolbox and Deep Learning Toolbox from Matlab are used.

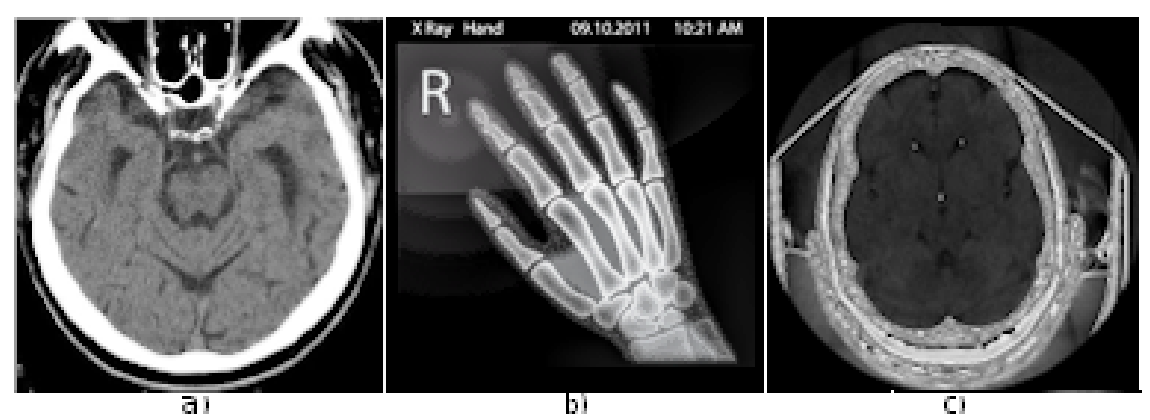

Figure 3. Data-set of medical images used for practical experiments: (a) Brain CT; (b) Hand X-ray [34], and (c) Head CT [35]. 

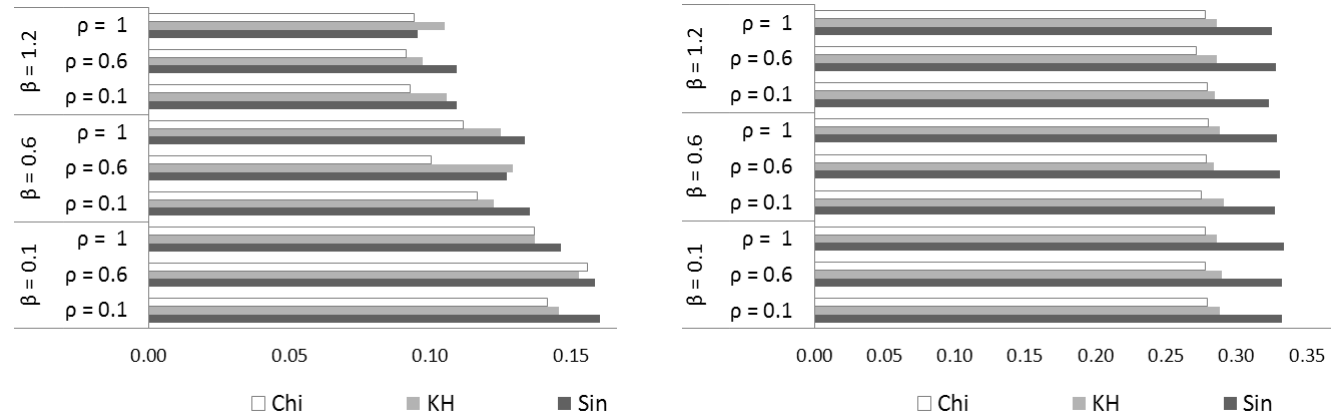

Figure 4. Parameters testing for Ant Colony Optimization (ACO) when $\alpha=1, \beta \in\{0.1,0.6,1.2\}$, $\rho \in\{0.1,0.6,1.0\}$ and $\psi=0.5$ (left) $\psi=0.001$ (right) for all considered operators Sin, KH and Chi; the $x$-coordinate represents the standardized value of correctly identified edges, with values in the unit interval.

Table 1. The standardized value of correctly identified edges with values in the unit interval for the medical images Brain CT, Hand X-ray and Head CT with every considered operator on ACO.

\begin{tabular}{cccc}
\hline Operator/Image & Brain_CT & Hand X-ray & Head_CT \\
\hline Ant Colony Optimization & & & \\
\hline Sin & 0.2933 & 0.2942 & 0.3302 \\
KH & 0.2932 & 0.2539 & 0.2832 \\
Chi & 0.2782 & 0.2450 & 0.2788 \\
\hline
\end{tabular}

\subsection{Representation of Results}

The following is the representation of medical images provided with identified edges on the data-set of medical images using each considered operator from Equations (16)-(18). Figures 5-7 show respectively the Brain CT, Hand X-ray and Head CT results of all considered operators when using Ant Colony Optimization before and after Denoise Convolutional Neural Network (DnCNN).

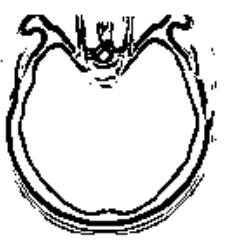

a)

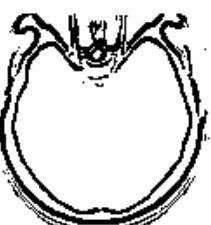

b)

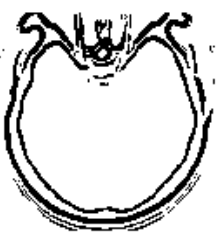

c)

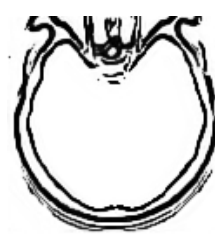

a)

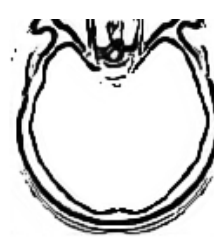

b)

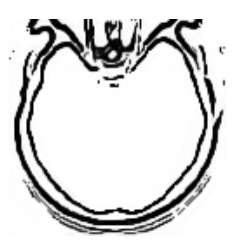

c)

Figure 5. Identified edges of Brain CT medical image using ACO (left) and ACO with Denoise Convolutional Neural Network (DnCNN) (right) (a) Sin operator Equation (16), (b) KH operator Equation (17), (c) Chi operator Equation (18).

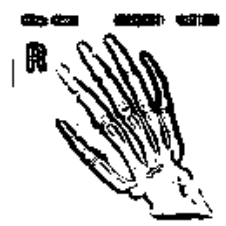

a)

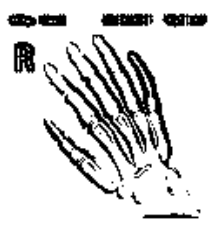

b)

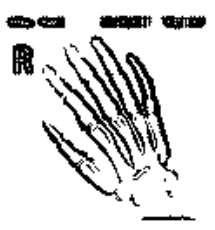

c)

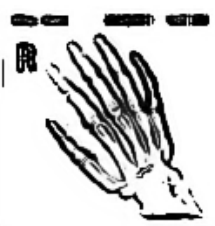

a)

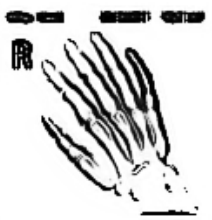

b)

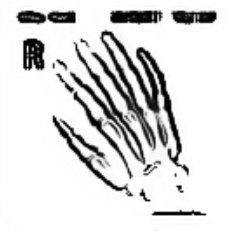

c)

Figure 6. Identified edges of Hand X-ray medical image using ACO (left) and ACO with DnCNN (right) with operators: (a) Sin operator Equation (16), (b) KH operator Equation (17), (c) Chi operator Equation (18). 

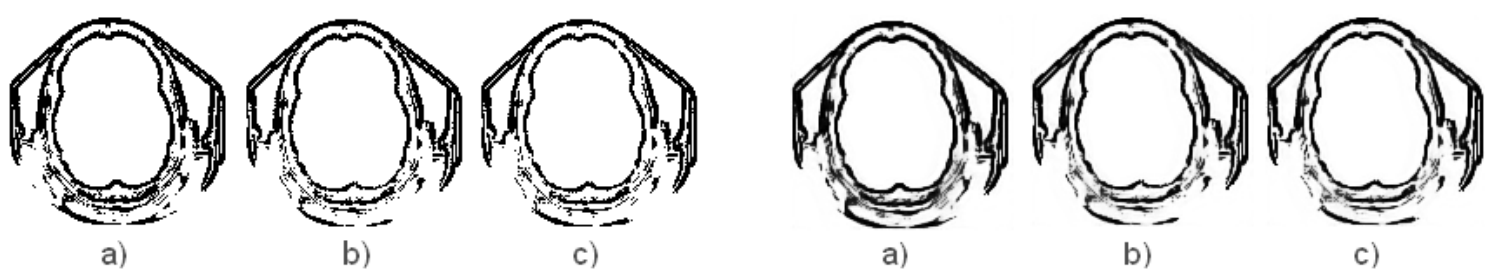

Figure 7. Identified edges of Head CT medical image using ACO (left) and ACO with DnCNN (right) with operators: (a) Sin operator Equation (16), (b) KH operator Equation (17), (c) Chi operator Equation (18).

\subsection{Discussions}

The main purpose of the current work is to study how the proposed operators influence the results of the edge detection problem; thus, the impact during ACO solver is discussed. Furthermore the influence of using the Denoise Convolutional Neural Network (DnCNN) for the quality of the final medical images is presented.

Table 2 with the results of ACO and its specified operators when image edge detection problem is solved shows the following: the highest values for means, 0.3059 is obtained for Sin and the same for the lowest best value 0.2933 . Nevertheless, ACO for edge detection shows its robustness as with the Chi operator provides the lowest values for deviations results: standard deviations 0.0193 for the average absolute deviation from median 0.0113 . The $K H$ operator shows stability during testing.

Table 2. Statistical values of the standardized value of correctly identified edges, with values in the unit interval, based on Table 1: mean, median, standard deviation (Std.Dev.), maximal (High), minimal (Low) and Average Absolute Deviation from median (Ave.Dev.) of overall results for ACO considering operators $\operatorname{Sin}$ Equation (16), proposed $\mathrm{KH}$-operator Equation (17) and proposed operator Chi-operator Equation (18).

\begin{tabular}{lcccccc}
\hline & Mean & Std.Dev. & High & Low & Median & Ave.Dev. \\
\hline ACO-Sin & 0.3059 & 0.0211 & 0.3302 & 0.2933 & 0.2942 & 0.0123 \\
ACO-KH & 0.2768 & 0.0204 & 0.2932 & 0.2539 & 0.2832 & 0.0131 \\
ACO-Chi & 0.2673 & 0.0193 & 0.2788 & 0.2450 & 0.2782 & 0.0113 \\
\hline
\end{tabular}

In order to compare ACO operators' results we make use of a similarity test. The higher similarity is shown by the smaller value. As similarity, the norm of images differences, the norm() function from MatLab is considered. Table 3 and Figure 8 illustrate similarities when comparing the similarities between ACO operators: Sin, KH and Chi.

The most similar operators are $\mathrm{Ch} i$ and $\mathrm{KH}$-operator with the two lowest values out of the three medical image results, 0.0726 for Hand X-ray and 0.0900 for Head CT. Less similar operators are Sin and $K H$.

Table 4 and Figure 9 illustrate the medical image similarities within the initial ACO methods and after using the pretrained Denoise Convolutional Neural Network (DnCNN) [37].

The most efficient operator, Chi, obtains the least changed image after DnCNN for Brain CT, 0.0612 value. The less efficient in this case was the Sin operator for Head CT, 0.0808 .

Table 3. Similarity comparison of the standardized value of correctly identified edges for medical images (Brain CT, Hand X-ray and Head CT with Sin, KH and Chi operators on ACO.

\begin{tabular}{cccc}
\hline Operator/Image & Brain_CT & Hand X-ray & Head_CT \\
\hline Ant Colony Optimization & & & \\
\hline KH - Chi & 0.1047 & $\mathbf{0 . 0 7 2 6}$ & $\mathbf{0 . 0 9 0 0}$ \\
Sin - Chi & 0.1090 & 0.1773 & 0.1774 \\
Sin - KH & $\mathbf{0 . 0 8 6 7}$ & 0.1640 & 0.1745 \\
\hline
\end{tabular}




\begin{tabular}{|c|c|c|c|}
\hline KH-Chi & 0.1047 & 0.0726 & 0.0900 \\
\hline $\operatorname{Sin}-K H$ & 0.0867 & 0.1640 & 0.1745 \\
\hline $\mathrm{Sin}-\mathrm{Chi}$ & 0.1090 & 0.1773 & 0.1774 \\
\hline
\end{tabular}

Figure 8. Similarities between ACO operators Sin, $\mathrm{KH}$ and $\mathrm{Chi}$ for medical images: (Brain CT, Hand X-ray and Head CT.

Table 4. Similarity comparison of the standardized value of correctly identified edges for medical images (Brain CT, Hand X-ray and Head CT with Sin, KH and Chi operators on ACO, before and after Denoise Convolutional Neural Network (DnCNN).

\begin{tabular}{cccc}
\hline Operator/Image & Brain_CT & Hand X-ray & Head_CT \\
\hline Ant Colony Optimization & & & \\
\hline Sin - Sin_DnCNN & 0.0670 & $\mathbf{0 . 0 7 4 8}$ & 0.0808 \\
KH - KH_DnCNN & 0.0668 & 0.0762 & 0.0719 \\
Chi - Chi_DnCNN & $\mathbf{0 . 0 6 1 2}$ & 0.0770 & $\mathbf{0 . 0 7 1 2}$ \\
\hline
\end{tabular}

\begin{tabular}{|c|c|c|c|}
\hline Chi-Chi\&DnCNN & 0.0612 & 0.0762 & 0.0712 \\
\hline KH-KH\&DnCNN & 0.0668 & 0.0748 & 0.0719 \\
\hline $\operatorname{Sin}-\operatorname{Sin} \& D n C N N$ & 0.0670 & 0.0770 & 0.0808 \\
\hline
\end{tabular}

Figure 9. Similarities before and after using the pretrained Denoise Convolutional Neural Network (DnCNN) [37] for all considered operators Sin, KH and Chi Brain CT, Hand X-ray and Head CT.

Table 5 shows the ranks of operators when using Ant Colony Optimization to identify medical edges and the most similar are $\mathrm{KH}$ and $\mathrm{Chi}$.

Table 5. Similarity ranks comparison of the standardized value of correctly identified edges with values in the unit interval for the medical images (Brain CT, Hand X-ray and Head CT with Sin, KH and Chi operators on Ant Colony Optimization using the norm diference, before and after using Denoise Convolutional Neural Network (DnCNN) [37].

\begin{tabular}{llll}
\hline \multicolumn{2}{l}{ Similarities Rank } & & \\
\hline ACO & ACO with DnCNN & \\
\hline $\boldsymbol{K H}, \boldsymbol{C h i}$ & rank 1: most similar & Chi-Chi_DnCNN & rank 1: most similar \\
Sin, $\mathbf{C h i}$ & rank 2 & KH - KH_DnCNN & rank 2 \\
Sin, $\mathbf{K H}$ & rank 3: less similar & Sin - Sin_DnCNN & rank 3: less similar \\
\hline
\end{tabular}

As the results suggested, the main advantages of the operators are the following:

- The Chi operator has the results similar with the Sin operator, so between Chi and KH, Chi operator performs better for image edge detection with ACO (Table 5).

- Chi operator provides the best results for standard deviations and the average absolute deviation from median when compared with Sin and KH operators The KH operator keeps its stability during all the tests including with DnCNN (Tables 2 and 5). 
- The best paper results is that the newly introduced operators $\mathrm{KH}$ and $\mathrm{Chi}$ preserve better the edges of the medical image during the image denoising process with DnCNN (Table 5).

Overall, the medical images edges obtained with the considered ACO operators shows reliability.

\section{Conclusions}

The health of people will be always a priority. In order to find out faster and with a high precision bone injuries within medical images, frequently made through X-rays, different types of software were implemented. Here, the meta-heuristic Ant Colony Optimization, ACO is used to identify edges of medical images. The current study introduces two new operators. One of the proposals is the $\mathrm{KH}$-operator based on the Krasnoselskij admissible perturbation of a demicontractive operator. The other one, is the admissible perturbation operator, the $C h i, \chi$-operator obtained by applying a function $\chi$ to an demicontractive mapping as specified by Rus in his work [1].

The use of different operators enhance the solution of the image edge detection problem. The motivation of the study was to see the behavior of demicontractive operators in edge detection algorithms, in particular with ACO. The results show the beneficent stability of the ACO operators and especially when using Denoise Convolutional Neural Network, DnCNN, to obtain medical images. An analysis is provided for both perspectives based on the imaged edge detection problem results: from operators perspective for ACO with and without using DnCNN denoise technique.

The promising results of the proposed operators could further empower other techniques, in order to identify better solutions not only just for medical image edge detection but as well as for other similar difficult problems. The study hopefully opens new directions for further studies in this area.

Author Contributions: Conceptualization C.T. and C.-M.P.; validation C.T., C.-M.P. and I.Z.; writing-original draft preparation C.T. and C.-M.P. and I.Z.; writing-review and editing C.T. and C.-M.P. All authors have read and agreed to the published version of the manuscript.

Funding: The research was funded by Technical University of Cluj-Napoca, Romania.

Conflicts of Interest: The authors declare no conflict of interest.

\section{Abbreviations}

The following abbreviations are used in this manuscript:

ACO Ant Colony Optimization

DnCNN Denoise Convolutional Neural Network

\section{References}

1. Rus, I.A. An abstract point of view on iterative approximation of fixed points. Fixed Point Theory 2012, 33, 179-192.

2. Berinde, V. Convergence theorems for fixed point iterative methods defined as admissible perturbations of a nonlinear operator. Carpathian J. Math. 2013, 29, 9-18.

3. Berinde, V.; Khan, A.R.; Fukhar-ud-din, H. Fixed point iterative methods defined as admissible perturbations of generalized pseudocontractive operators. J. Nonlinear Convex Anal. 2015, 16, 563-572.

4. Kitkuan, D.; Kumam, P.; Berinde, V.; Padcharoen, A. Adaptive algorithm for solving the SCFPP of demicontractive operators without a priori knowledge of operator norms. Ann. Univ. Ovidius Math. Ser. 2019, 27, 153-175. [CrossRef]

5. Padcharoen, A.; Kumam, P.; Cho, Y.J. Split common fixed point problems for demicontractive operators. Numer. Algorithms 2019, 82, 297-320. [CrossRef]

6. Wang, Y.; Fang, X.; Kim, T.W. Viscosity Methods and Split Common Fixed Point Problems for Demicontractive Mappings. Mathematics 2019, 7, 844. [CrossRef]

7. Wang, F.; Xu, H.K. Weak and strong convergence of two algorithms for the split fixed point problem. Numer. Math. Theory Method Appl. 2018, 11, 770-781. 
8. Ticala, C. Approximating solutions of generalized pseudocontractive variational inequalities by admissible perturbation type iterative methods. Creat. Math. Inf. 2013, 22, 237-241.

9. Ticala, C. A weak convergence theorem for a Krasnoselskij type fixed point iterative method in Hilbert spaces using an admissible perturbation. Sci. Stud. Res. 2015, 25, 243-252.

10. Ticala, C. Approximating fixed points of demicontractive mappings by iterative methods defined as admissible perturbations. Creat. Math. Inf. 2016, 25, 121-126.

11. Dorigo, M.; Stützle, T. Ant colony optimization: Overview and recent advances. In Handbook of Metaheuristics; Springer: Cham, Switzerland, 2019; pp. 311-351.

12. Dorigo, M.; Stŭtzle, T. Ant Colony Optimization; MIT Press: Cambridge, MA, USA, 2004.

13. Chira, C.; Pintea, C.M.; Dumitrescu, D. Learning sensitive stigmergic agents for solving complex problems. Comput. Inform. 2010, 29, 337-356.

14. Pintea, C.-M.; Pop, P.C.; Chira, C.; Dumitrescu, D. A Hybrid Ant-Based System for Gate Assignment Problem. Lect. Notes Comp. Sci. 2008, 5271, 273-280.

15. Crisan, G.C.; Nechita, E.; Palade, V. Ant-based system analysis on the traveling salesman problem under real-world settings. In Combinations of Intelligent Methods and Applications; Springer: Cham, Switzerland, 2016; pp. 39-59.

16. Bella, J.E.; McMullen, P.R. Ant colony optimization techniques for the vehicle routing problem Adv. Eng. Inf. 2004, 18, 41-48. [CrossRef]

17. Pintea, C.M.; Pop, P.C.; Dumitrescu, D. An ant-based technique for the dynamic generalized travelling salesman problem. In Proceedings of the 7th International Conference on Systems Theory and Scientific Computation, Athens, Greece, 24-26 August 2007; pp. 255-259.

18. Zhang, D.Y.; Zhao, H. Research of Generalized Ant Colony Algorithm for Logistics and Distribution. Appl. Mech. Mater. 2014, 513-517, 691-694. [CrossRef]

19. Pintea, C.-M.; Pop, P.C.; Chira, C. The generalized traveling salesman problem solved with ant algorithms. Complex Adapt. Syst. Model. 2017, 5, 8. [CrossRef]

20. Chowdhury, S.; Marufuzzaman, M.; Tunc, H.; Bian, L.; Bullington, W. A modified Ant Colony Optimization algorithm to solve a dynamic traveling salesman problem: A case study with drones for wildlife surveillance. J. Comput. Des. Eng. 2019, 6, 368-386. [CrossRef]

21. Shi, Q.; An, J.; Gagnon, K.K.; Cao, R.; Xie, H. Image Edge Detection Based on the Canny Edge and the Ant Colony Optimization Algorithm. In Proceedings of the International Congress on Image and Signal Processing, BioMedical Engineering and Informatics (CISP-BMEI), Suzhou, China, 19-21 October 2019; pp. 1-6.

22. Mahalingam, T.; Subramoniam, M. ACO-MKFCM: An Optimized Object Detection and Tracking Using DNN and Gravitational Search Algorithm. Wirel. Pers. Commun. 2020, 110, 1567-1604. [CrossRef]

23. Yu, Y.; Deng, Q.; Ren, L.; Tashi, N. Memristor Crossbar Array Based ACO for Image Edge Detection. Neural Process Lett. 2020, 51, 1891-1905. [CrossRef]

24. Rundo, L.; Militello, C.; Vitabile, S.; Russo, G.; Sala, E.; Gilardi, M.C. A Survey on Nature-Inspired Medical Image Analysis: A Step Further in Biomedical Data Integration. Fundam. Inf. 2020, 171, 345-365. [CrossRef]

25. Lahrichi, N.; Crainic, T.G.; Gendreau, M.; Rei, W.; Crişan, G.C.; Vidal, T. An integrative cooperative search framework for multi-decision-attribute combinatorial optimization: Application to the MDPVRP. Eur. J. Oper. Res. 2015, 246, 400-412. [CrossRef]

26. Pintea, C.M.; Chira, C.; Dumitrescu, D.; Pop, P. A sensitive metaheuristic for solving a large optimization problem. Lect. Notes Comput. Sci. 2008, 4910, 551-559.

27. Pintea, C.-M.; Chira, C.; Dumitrescu, D. Sensitive Ants: Inducing Diversity in the Colony. Stud. Comput. Intell. 2009, 236, 15-24.

28. Pintea, C.-M.; Ticala, C. Medical image processing: A brief survey and a new theoretical hybrid ACO model. In Combinations of Intelligent Methods and Applications; Springer: Cham, Switzerland, 2016; pp. 117-134.

29. Ticala, C.; Zelina, I. New ant colony optimization algorithm in medical images edge detection. Creat. Math. Inf. 2020, 29, 101-108.

30. Hicks, T.L.; Kubicek, J.D. On the Mann iteration in a Hilbert space. J. Math. Anal. Appl. 1977, 59, $498-504$. [CrossRef]

31. Chidume, C.; Maruster, S. Iterative methods for the computation of fixed points of demicontractive mappings. J. Comput. Appl. Math. 2010, 234, 861-882. [CrossRef] 
32. Tian, J.; Yu, W.; Xie, S. An ant colony optimization algorithm for image edge detection. In Proceedings of the IEEE Congress on Evolutionary Computation (IEEE World Congress on Computational Intelligence), Hong Kong, China, 1-6 June 2008; pp. 751-756.

33. Otsu, N. A threshold selection method from gray-level histograms. IEEE Trans. Syst. Man Cybern. 1979, 9, 62-66. [CrossRef]

34. X-ray Hand. Vista Medical Pack. License: Free for Non Commercial Use. id, 236487. Available online: https: / / www.iconspedia.com/ (accessed on 1 May 2020).

35. Head CT. Online Medical Free Image. Available online: http://www.libpng.org/pub/png/pngvrml/ct2.9128x128.png (accessed on 1 May 2020).

36. Kanchi-Tian. Image Edge Detection Using Ant Colony Optimization. Version 1.2.0.0, Updated 24 January 2011. MATLAB Central File Exchange. Available online: https:/ / www.mathworks.com/matlabcentral/ fileexchange/20997-image-edge-detection-using-ant-colony-optimization (accessed on 1 May 2020).

37. Denoise Image Using Deep Neural Network. MATLAB Central File Exchange. Retrieved in 10 June. 2020. Available online: https://www.mathworks.com/help/images/ref/denoiseimage.html (accessed on 1 May 2020).

(C) 2020 by the authors. Licensee MDPI, Basel, Switzerland. This article is an open access article distributed under the terms and conditions of the Creative Commons Attribution (CC BY) license (http://creativecommons.org/licenses/by/4.0/). 